\title{
Bioanalysis
}

\section{Platforms and techniques used for biomarker assays: where are we now?}

\author{
"...the biomarker bioanalyst now has the tools that are critical to \\ address and resolve the unique biological questions and challenges \\ that exist over the course of the drug development process."
}

First draft submitted: 28 April 2017; Accepted for publication: 15 May 2017; Published online: 24 July 2017

\section{Keywords: biomarker $\bullet$ ligand binding assay $\bullet$ LC-MS $\bullet$ parallelism $\bullet$ platform $\bullet$ surrogate} analyte • surrogate matrix

Currently, the term biomarker has many connotations, as such, it is important to properly define this term for the context of this editorial. In the broadest definition, biomarkers are "a characteristic that is objectively measured and evaluated as an indicator of normal biological processes, pathogenic processes or pharmacological responses to a therapeutic intervention" [1]. However, in the context of biomarkers evaluated during the course of clinical drug trials, we can categorically divide the term into three categories which include biomarkers for determining risk (predisposition), biomarkers used for diagnosis (screening, prognosis and diagnosis) and biomarkers specific to pharmacology (predictive, target engagement, mechanism engagement, clinical engagement, surrogate and clinical end point).

As the number of drugs targeting a specific biological mechanism increases, the pharmacodynamic (PD) biomarkers involved in that mechanism can provide a valuable understanding of target engagement, efficacy and/or safety to assist in guiding the drug development process for that mechanism. When measuring PD biomarkers in samples from preclinical and/or clinical studies, it is important that the robustness and limitations of the analytical methods as well as stability of biomarker in the matrix of interest is well understood allowing for minimal analytical variation and lack of biomarker degradation during sample collection.
A variety of bioanalytical platforms are routinely applied to measurement of biomarker analytes, including: ligand-binding assays (LBA), LC-MS, flow cytometry and immunohistochemistry. Although each of these platforms can be used to understand the impact of a drug on PD end points, LBA and LC-MS are the two platforms most commonly utilized and will be the focus of this editorial. The most appropriate platform to monitor a given biomarker depends on the matrix, analyte and specific biological question to be answered. However, in some instances either platform could be used to support an analytical measurement, therefore understanding the biological question and/or problem and the benefits of a given platform should guide this selection. This editorial aims to address the challenges and benefits of either of these platforms in relation to the unique issues associated with specific biomarker analyte quantitation.

\section{Sensitive versus selective biomarker platforms}

As a number of biomarkers of interest are present in matrices at very low concentrations (i.e., cytokines), development of a highly sensitive assay is a critical component to platform selection. However, as the need to develop ever more sensitive assays increases, problems with developing selective assays becomes more challenging. Historically, photometric LBAs were the most commonly used platform for both commercial and bespoke assays, however,
Charles Hottenstein*,1, Matthew Szapacs'1, Kerensa Fuller $^{2}$ \& Christopher Evans ${ }^{1}$ ${ }^{1}$ GlaxoSmithKline, PTS - In Vivo/In Vitro Translation; Bioanalysis, Immunogenicity \& Biomarkers; 709 Swedeland Road, King of Prussia, PA 19406 USA ${ }^{2}$ GlaxoSmithKline, PTS - In Vivo/In Vitro Translation; Bioanalysis, Immunogenicity and Biomarkers; Gunnels Wood Road, Stevenage, Herts SG1 2NY, UK *Author for correspondence: charles.s.hottenstein@gsk.com 
the electrochemiluminescent format from Meso Scale Discovery (MSD) has over the past decade become the industry standard, with a large number of commercially available biomarker kits that can be used as is or optimized and validated for regulated use. Although the MSD platform offers improved selectivity and dynamic range over more traditional photometric methods, it represents a single vendor platform with proprietary instrumentation and microtiter plate labware. Where bespoke assays are developed, consistency in microtiter plate lotto-lot variability of the background and overall signal range have caused issues when changing microtiter plate lots for established methods. Therefore, the benefits and risks should be closely considered when choosing single vendor platforms for biomarker quantitation.

\section{"One of the most common challenges for biotherapeutics is the development of a free, total and/or complex target engagement assay.}

In addition, various vendors have been developing proprietary ultrasensitive instrumentation to allow quantitation of very low abundance biomarkers, including: MSD platform (S-Plex), Singulex and Quanterix Simoa with the various platforms providing different sensitivities depending on the analyte of interest $[2,3]$. Although these platforms can provide high sensitivity assays, they also suffer from additional challenges associated with selectivity from individual samples due to the low levels of analyte that are being detected and often lead to a trade-off between assay sensitivity and overall selectivity. It should be noted that the Singulex and Quanterix Simoa are also single-vendor platforms and the same considerations listed above for the MSD platform should apply when selecting these higher sensitivity instrument for use.

One platform that has emerged to address inherent selectivity challenges identified in LBA platforms is LC-MS/MS. Through the use of immunocapture reagents followed by enzymatic digestion and selection of a surrogate peptide which is coupled to LC-MS, we and others [4-6] have shown that this platform can be used to develop highly selective and sensitive assays utilizing immunocapture reagents that may not be suitable for use in a typical LBAs. In addition to mitigating the observed selectivity issues in LBAs, there are also occasions when the LC-MS platform may provide the more sensitive analytical option as the selectivity and overall S/N of the assay is improved; therefore platform selection needs to be made on a case-by-case basis.

As well as sensitivity, LBA formats often require less expensive specialized equipment and are often a more economical option than LC-MS platforms. In our laboratories, we employ all of the above described approaches for biomarker quantitation depending on the availability of reagents, while balancing the benefits of sensitivity and selectively when choosing one platform over another allowing one to accurately answer to biological question at hand.

\section{Measuring biomarkers in the presence of endogenous analyte}

Another common challenge encountered during quantitation of biomarkers is determination of the absolute concentration of a biomarker. Unlike pharmacokinetic methods, where the calibration matrix is typically analyte free, allowing for the detection of absolute drug concentrations; biomarker matrices often contain some level of endogenous analyte and the biomarker bioanalyst often relies on either a depleted matrix approach to provide analyte free matrices for calibration purposes or the use of low- and high-concentration megapools with subsequent admixing of these pools to create a uniform control and potentially use for calibration purposes [7].

However, both approaches are not without issue as depleting the matrix may remove other components and the megapools often are not available for novel or less well-studied biomarkers. With this in mind, the use of surrogate matrix or surrogate analyte strategies to generate a standard calibration curve without interference from endogenous biomarkers continues to be standard practice. Surrogate matrix strategies are the most common approaches and use buffer with added proteins like bovine serum albumin, commercially available matrices (SeraSub, UriSub) or other in-house mixtures of proteins in an aqueous buffer all with spiked analyte. The use of surrogate matrix requires that a parallelism assessment is completed by spiking analyte in both surrogate matrix and authentic matrix across the assay range. If the surrogate matrix curve is 'parallel' to the authentic matrix curve then the surrogate matrix is representative of the authentic matrix [8].

As LC-MS continues to grow as a platform for biomarker bioanalytical support, the use of a surrogate analyte approach has become more commonplace. Surrogate analyte methodologies utilize an isotopically labeled biomarker that can be used to create a standard calibration curve in the authentic matrix as it has a unique mass that can be differentiated from the authentic, endogenous analyte using LC-MS [9]. This technique has enormous promise for development of biomarker assays as the authentic matrix that is identical to that of the samples can be used for study support and allow for absolute quantitation. In addition, the use of longer gradients or high-resolution LC-MS provides increased confidence and confirmation of the endogenous analyte being measured and demonstrates that it is not a cross-reactive species or a molecule with a similar mass transition in the MS. 


\section{Measuring the various forms of biomarker targets}

One of the most common challenges for biotherapeutics is the development of a free, total and/or complex target engagement assay. Traditionally, LBA-based platforms have been used for the quantitation of free, total or complex target engagement and have been reviewed previously [10]. However, the ex vivo manipulation of samples required before analysis (i.e., sample dilution, extraction, etc.) can cause an equilibrium bias between free, total and complex forms in solution. Therefore, the development of assays that provide 'true' free, total and/or complex measurements is quite challenging and the assays often do not demonstrate agreement when used in combination.

In our laboratories, we prefer the combination of total and complex assay measurements to understand biomarker PDs in relation to the administered drug and feel that free assays are the most challenging and often generate less robust data for a number of reasons including equilibrium shifts from native sample matrix upon dilution, the density of the solid phase capture mechanism concentration compared with analyte concentration in solution and the length of primary incubation times. Typically, we avoid free assays unless the methods are highly characterized and the quality of the data can be assured. Overall, drug target complex and total target assays are less susceptible to these issues and have been shown to demonstrate the best measure of target engagement and target concentration [11].

\section{References}

1 Atkinson AJ, Colburn WA, Degruttola VG, Demets DL. Surrogate endpoints: preferred definitions and conceptual framework* biomarkers and surrogate endpoints: preferred definitions and conceptual framework. Clin. Pharmacol. Ther. 69(3), 89-95 (2001).

2 Oberoi P, Suschak J, Stenglin M et al. True sensitivity of immunoassays: are concentrations of low abundance analytes real or artifacts? J. Immunol. 196(Suppl. 1), 138.139 (2016).

3 Fischer SK, Joyce A, Spengler M et al. Emerging technologies to increase ligand binding assay sensitivity. AAPS J. 17(1), 93-101 (2015).

4 Bronsema KJ, Bischoff R, Pijnappel WP, van der Ploeg AT, van de Merbel NC. Absolute quantification of the total and antidrug antibody-bound concentrations of recombinant human $\alpha$-glucosidase in human plasma using protein $\mathrm{G}$ extraction and LC-MS/MS. Anal. Chem. 87(8), 4394-4401 (2015).

5 Palandra J, Finelli A, Zhu M, Masferrer J, Neubert H. Highly specific and sensitive measurements of human and monkey interleukin 21 using sequential protein and tryptic peptide immunoaffinity LC-MS/MS. Anal. Chem. 85(11), 5522-5529 (2013).

\section{Conclusion}

The best-suited quantitation platform for a given biomarker measurement depends on a number of factors including the required sensitivity, selectivity with the ability to use the actual matrix and actual biomarker analyte or will a surrogate or combination approach need to be used. The ability to select from more than one analytical option in many situations is beneficial for the biomarker bioanalyst and allows for the ability to proactively address analytical issues allowing for high-quality reliable data for internal or pivotal decision-making purposes regarding the safety or efficacy of a drug candidate over the course of the drug development process. With the recent bioanalytical advancements and ability to couple platforms (hybrid LBA/LC-MS), the biomarker bioanalyst now has the tools that are critical to address and resolve the unique biological questions and challenges that exist over the course of the drug development process.

\section{Financial \& competing interests disclosure}

The authors have no relevant affiliations or financial involvement with any organization or entity with a financial interest in or financial conflict with the subject matter or materials discussed in the manuscript. This includes employment, consultancies, honoraria, stock ownership or options, expert testimony, grants or patents received or pending, or royalties.

No writing assistance was utilized in the production of this manuscript.

6 Ackermann BL, Berna MJ. Coupling immunoaffinity techniques with MS for quantitative analysis of lowabundance protein biomarkers. Expert Rev. Proteomics 4(2), 175-186 (2007).

7 Arnold ME, Booth B, King L, Ray C. Workshop Report: Crystal City VI - Bioanalytical Method Validation for Biomarkers. AAPS J. 18(6). 1366-1372 (2016).

8 Jones BR, Schultz GA, Eckstein JA, Ackermann BL. Surrogate matrix and surrogate analyte approaches for definitive quantitation of endogenous biomolecules. Bioanalysis 4(19), 2343-2356 (2012).

9 Jenkins RG. Accuracy: a potential quandary in regulated bioanalysis of 'endogenous' analytes. Bioanalysis 8(23), 2393-2397 (2016).

10 Talbot JJ, Calamba D, Pai M, Ma M, Thway TM. Measurement of free versus total therapeutic monoclonal antibody in pharmacokinetic assessment is modulated by affinity, incubation time, and bioanalytical platform. AAPS J. 17(6), 1446-1454 (2015).

11 Lee JW, Kelley M, King LE et al. Bioanalytical approaches to quantify "total" and "free" therapeutic antibodies and their targets: technical challenges and PK/PD applications over the course of drug development. AAPS J. 13(1), 99-110 (2011). 